PROCEEDINGS OF THE

AMERICAN MATHEMATICAL SOCIETY

Volume 129, Number 11, Pages 3345-3351

S 0002-9939(01)05955-X

Article electronically published on April 2, 2001

\title{
A NICELY BEHAVED SINGULAR INTEGRAL ON A PURELY UNRECTIFIABLE SET
}

\author{
PETRI HUOVINEN
}

(Communicated by David Preiss)

Abstract. We construct an example of a purely 1-unrectifiable AD-regular set $E$ in the plane such that the limit

$$
\lim _{r \downarrow 0} \int_{E \backslash B(x, r)} K(x-y) d \mathcal{H}^{1}(y)
$$

exists and is finite for $\mathcal{H}^{1}$ almost every $x \in E$ for some class of antisymmetric Calderón-Zygmund kernels. Moreover, the singular integral operators associated with these kernels are bounded in $L^{2}(F)$, where $F \subset E$ has a positive $\mathcal{H}^{1}$ measure.

\section{INTRODUCTION}

In this note "a nice behaviour of singular integral operator" in the complex plane is considered in two ways: in the sense of " $L^{2}$-boundedness" and in the sense of "existence of principal values". A singular integral operator $T$ associated with a kernel $K$ is bounded in $L^{2}(E)$ if there exists $M<\infty$ such that

$$
\int_{E}\left|\int_{E \backslash B(z, \varepsilon)} g(y) K(x-y) d \mathcal{H}^{1}(y)\right|^{2} d \mathcal{H}^{1}(x) \leq M \int_{E}|g|^{2} d \mathcal{H}^{1}
$$

for all $g \in L^{2}(E)$ and $\varepsilon>0$, where $K: \mathbb{C} \backslash\{0\} \rightarrow \mathbb{C}$ is a standard kernel, as in [2]. We say that the principal values for the singular integral associated with the kernel $K$ and a set $E$ exist $\mathcal{H}^{1}$ almost everywhere if

$$
\lim _{\varepsilon \downarrow 0} \int_{E \cap\{y:|x-y| \geq \varepsilon\}} K(x-y) d \mathcal{H}^{1}(y)
$$

exists and is finite for $\mathcal{H}^{1}$ almost every $x \in E$.

We will also use the following concepts to describe geometric properties of sets. A set $E$ is Ahlfors-David regular (AD-regular) if there is $0<M_{0}<\infty$ such that for all $z \in E$ and $0<r<\operatorname{diam}(E)$,

$$
r / M_{0} \leq \mathcal{H}^{1}(E \cap\{x \in \mathbb{C}:|z-x|<r\}) \leq M_{0} r .
$$

Received by the editors August 31, 1999 and, in revised form, March 22, 2000.

2000 Mathematics Subject Classification. Primary 28A75, 42B20; Secondary 30E20.

Key words and phrases. Singular integrals, rectifiability.

The author was supported by EU TMR Grant \#ERBFMBICT972410. 
A set $E$ with $\mathcal{H}^{1}(E)<\infty$ is called purely 1-unrectifiable if it intersects any Lipschitz curve in a set of $\mathcal{H}^{1}$ measure zero.

The question as to whether singular integrals behave nicely on a set that is in some sense rectifiable has been an object of investigation for over twenty years. Calderón proved in 1 the $L^{2}$-boundedness of the Cauchy singular integral operator on a Lipschitz graph with a small Lipschitz constant. Later this result was extended to other kernels and a wider class of rectifiable sets. For a survey of the results of this type see [6] or [7, Chapter 20].

The converse question of whether the nice behaviour of singular integrals implies some kind of rectifiability has also long been a topic of investigations. However, most of the work has been done for the Cauchy singular integral. This is mainly because of the importance of the Cauchy integral itself and its application to the problem of removability of bounded analytic functions (see e.g. [8], [5] and 4]), but also because extending the theory seems to be difficult.

In this paper we give examples of singular integral operators in the plane which behave nicely both on 1-rectifiable sets (see e.g. [7, Chapter 20] for results of this type) and also on some purely 1-unrectifiable sets. In fact, we construct a purely 1-unrectifiable $\mathrm{AD}$-regular set $E \subset \mathbb{C}$ for which principal values exist $\mathcal{H}^{1}$ almost everywhere. Moreover, we prove $L^{2}$-boundedness in a subset of the original set. To prove the last fact we use [10, Theorem 2.2], although in this case it would be easy to show $L^{2}$-boundedness directly or using a dyadic version of $T(b)$-theorem (see [2]).

\section{Preliminaries}

First we introduce some notation. For $a \in \mathbb{C}, 0<r<R<\infty$ and $A \subset \mathbb{C}$, we let

$$
\begin{aligned}
B(a, r) & =\{z \in \mathbb{C}:|z-a| \leq r\}, \\
A(x, r, R) & =\{z \in \mathbb{C}: r<|z-a| \leq R\}, \\
\operatorname{dist}(a, A) & =\inf \{|z-a|: z \in A\} .
\end{aligned}
$$

Also, let $\partial A$ denote the boundary of $A$, and define $\operatorname{diam}(A)$ to be the diameter of $A$. By a Borel measure we mean a non-negative measure on the Borel $\sigma$-algebra and $\mathcal{H}^{1}$ stands for the 1-dimensional Hausdorff measure. If $\mu$ is a Borel measure, then the restriction of $\mu$ to a Borel set $A, \mu\llcorner A$, is defined by $(\mu \mathrm{L} A)(B)=\mu(A \cap B)$.

A kernel $K: \mathbb{C} \backslash\{0\} \rightarrow \mathbb{C}, K \neq 0$, is an antisymmetric function which satisfies the following four conditions for some $0<C<\infty$ and for all $x \in \mathbb{C} \backslash\{0\}, y \neq x$, $z \neq x$ and $r>0$ :

$$
\begin{aligned}
|K(x-y)-K(x-z)| & \leq C \frac{|y-z|}{|x-y||x-z|}, \\
|K(x)| & \leq C \frac{1}{|x|} \\
K(r) & =K(-r)=0 \\
K(x) & =-K(-\bar{x}) .
\end{aligned}
$$

We note that such a class of kernels is non-empty because it contains, for instance, the kernel $K(z)=\operatorname{Re}(z) /|z|^{2}-\operatorname{Re}(z)^{3} /|z|^{4}$. The conditions (2.1) and (2.2) are commonly used for standard kernels and, for example, the Cauchy kernel satisfies them. However, the requirements (2.3) and (2.4) are special and will guarantee the 
good behaviour of singular integral operators associated with the kernels that are needed to prove the result.

To estimate the integrals of the kernel, we will use the norms

$$
\|f\|_{A}=\sup _{x \in A}|f(x)| \text { and }\|f\|=\|f\|_{\mathbb{C}}
$$

of functions $f: \mathbb{C} \rightarrow \mathbb{C}$ and the distance

$$
d(\mu, \nu)=\sup \left\{\left|\int f d \mu-\int f d \nu\right|: \operatorname{Lip}(f) \leq 1\right\}
$$

of Borel probability measures $\mu, \nu$ in $\mathbb{C}$ with compact support. We note that $d$ restricted to the set of measures with support contained in a fixed compact set $K \subset \mathbb{C}$ is equivalent to the distances $F_{D}(\mu, \nu)$ from [7, Chapter 14] if $D$ is sufficiently large; in particular, on this set $d$ is a complete metric metrizing the weak topology.

Lemma 2.1. If $\mu, \nu$ are Borel probability measures in $\mathbb{C}, A \subset \mathbb{C}$ is a compact set and $t>0$, then

$$
\mu(A) \leq \nu\{x: \operatorname{dist}(x, A)<t\}+d(\mu, \nu) / t
$$

and, if $h: A \rightarrow \mathbb{C}$ is a Lipschitz function, then

$$
\left|\int_{A} h d \mu-\int_{A} h d \nu\right| \leq\left(\operatorname{Lip}(h)+4\|h\|_{A} / t\right) d(\mu, \nu)+2\|h\|_{A} \nu\{x: \operatorname{dist}(x, \partial A)<t\} .
$$

Proof. For the first statement define $\psi(x)=\max (0,1-\operatorname{dist}(x, A) / t)$ and estimate $\mu(A) \leq \int \psi d \mu \leq \int \psi d \nu+d(\mu, \nu) \operatorname{Lip}(\psi) \leq \nu\{x: \operatorname{dist}(x, A)<t\}+d(\mu, \nu) / t$.

For the second statement define $\varphi(x)=h(x)$ if $x \in A, \varphi(x)=0$ if $\operatorname{dist}(x, A)>$ $t / 2$, and extend $\varphi$ to the whole complex plane using Kirszbraun's theorem so that $\|\varphi\| \leq\|h\|_{A}$ and $\operatorname{Lip}(\varphi) \leq \max \left(\operatorname{Lip}(h), 2\|h\|_{A} / t\right)$. Using also the first statement, we get

$$
\begin{aligned}
\left|\int_{A} h d \mu-\int_{A} h d \nu\right| \leq & \left|\int \varphi d \mu-\int \varphi d \nu\right|+\int_{C \backslash A}|\varphi| d \mu+\int_{C \backslash A}|\varphi| d \nu \\
\leq & \operatorname{Lip}(\varphi) d(\mu, \nu)+\|h\|_{A} \mu\{x: 0<\operatorname{dist}(x, A)<t / 2\} \\
& +\|h\|_{A} \nu\{x: 0<\operatorname{dist}(x, A)<t / 2\} \\
\leq & \left(\operatorname{Lip}(h)+4\|h\|_{A} / t\right) d(\mu, \nu) \\
& +2\|h\|_{A} \nu\{x: \operatorname{dist}(x, \partial A)<t\} .
\end{aligned}
$$

\section{The construction of the example}

In this section we give the promised example of an AD-regular set $E$ which is purely unrectifiable and for which principal values exist $\mathcal{H}^{1}$ almost everywhere for all kernels satisfying our conditions.

Theorem 3.1. There exists a purely unrectifiable compact AD-regular set $E \subset \mathbb{C}$ such that $\mathcal{H}^{1}(E)>0$ and for $\mathcal{H}^{1}$ almost every $x \in E$, the limit of the integral in (1.2) exists and is finite with any kernel $K$ satisfying conditions (2.1) -(2.4).

Proof. The set $E$ will be obtained as the limit of a sequence of sets $E_{n}$ with each $E_{n}$ being the union of a set $\mathcal{I}_{n}$ of line segments parallel to the real axis. We first describe a recursive construction of the families $\mathcal{I}_{n}$. Let $\left(m_{k}\right)$ be a sufficiently quickly increasing sequence of positive integers starting with $m_{0}=1$; for example, 
the condition $m_{k+1}>\left(16^{k+4} m_{0} m_{1} \cdots m_{k}\right)^{2}$ will be sufficient for all our purposes. We let $\mathcal{I}_{0}=\{[0,1]\}$. Whenever $\mathcal{I}_{n-1}$ has been defined and $n$ is odd, we let

$$
\mathcal{I}_{n}=\mathcal{I}_{n-1} \cup\left\{i \operatorname{diam}(J) / m_{n}+J: J \in \mathcal{I}_{n-1}\right\} \text {, where } i=\sqrt{-1},
$$

and, if $n$ is even, we denote for each $J=[x, y] \in \mathcal{I}_{n-1}$ and $l=1,2, \ldots, m_{n}$,

and define

$$
J_{l}=\left[x+\frac{2 l-1}{2 m_{n}}(y-x), x+\frac{2 l}{2 m_{n}}(y-x)\right]
$$

$$
\mathcal{I}_{n}=\left\{J_{l}: J \in \mathcal{I}_{n-1}, l=1, \ldots, m_{n}\right\}
$$

Now set $E_{n}:=\bigcup_{J \in \mathcal{I}_{n}} J$. Denote by $\lambda_{n}$ the length of the intervals in $\mathcal{I}_{n}$; so $\lambda_{2 n}=$ $\lambda_{2 n+1}=2^{-n}\left(m_{0} m_{2} \cdots m_{2 n}\right)^{-1}$. We also note that the minimal distance of two different intervals from $\mathcal{I}_{n}$ is $d_{n}=\lambda_{n}$ if $n$ is even and $d_{n}=\lambda_{n} / m_{n}$ if $n$ is odd, and observe that $\operatorname{dist}\left(x, E_{n}\right) \leq d_{n}$ for each $x \in E_{n-1}$ and $\operatorname{dist}\left(x, E_{n-1}\right) \leq d_{n}$ for each $x \in E_{n}$. By definition, the Hausdorff distance of $E_{n}$ and $E_{n-1}$ is

$$
\operatorname{dist}\left(E_{n}, E_{n-1}\right)=\max \left(\max _{x \in E_{n}} \operatorname{dist}\left(x, E_{n-1}\right), \max _{x \in E_{n-1}} \operatorname{dist}\left(x, E_{n}\right)\right) \leq d_{n} .
$$

Since $\sum_{k} d_{k}<\infty$ and the space of non-empty compact subsets of $\mathbb{C}$ equipped with the Hausdorff metric is complete, the sequence $E_{n}$ converges in the Hausdorff metric to a compact set $E$ and $\operatorname{dist}\left(E, E_{n}\right) \leq \sum_{k=n+1}^{\infty} d_{k} \leq 2 d_{n+1}$. To obtain the last inequality, we used that $d_{k+1} \leq d_{k} / 2$; in fact, the sequence $m_{k}$ increases so quickly that

$$
d_{k+1} \leq 4^{-k} d_{k}^{2}
$$

We define

$$
\mu_{n}= \begin{cases}\frac{1}{2} \mathcal{H}^{1}\left\llcorner E_{n}\right. & \text { for odd } n, \\ \mathcal{H}^{1}\left\llcorner E_{n}\right. & \text { for even } n .\end{cases}
$$

Then $\mu_{n}$ are Borel probability measures in $\mathbb{C}$ and, for any Lipschitz function $f$,

$$
\begin{aligned}
\left|\int f d \mu_{n}-\int f d \mu_{n-1}\right| & \leq \sum_{J \in \mathcal{I}_{n-1}} \sum_{l=1}^{m_{n}} \int_{J_{l}}\left|f\left(x-d_{n}\right)-f(x)\right| d \mathcal{H}^{1}(x) \\
& \leq \operatorname{Lip}(f) d_{n} \sum_{J \in \mathcal{I}_{n-1}} \sum_{l=1}^{m_{n}} \mathcal{H}^{1}\left(J_{l}\right)=\operatorname{Lip}(f) d_{n} / 2
\end{aligned}
$$

if $n$ is even, and

$$
\begin{aligned}
\left|\int f d \mu_{n}-\int f d \mu_{n-1}\right| & \leq \sum_{J \in \mathcal{I}_{n-1}} \int_{J}\left|f\left(x+i d_{n}\right)-f(x)\right| d \mathcal{H}^{1}(x) / 2 \\
& \leq \operatorname{Lip}(f) d_{n} \sum_{J \in \mathcal{I}_{n-1}} \mathcal{H}^{1}(J) / 2=\operatorname{Lip}(f) d_{n} / 2
\end{aligned}
$$

if $n$ is odd. Hence $d\left(\mu_{n}, \mu_{n-1}\right) \leq d_{n} / 2$. As $\sum_{k} d_{k}<\infty$ and the union of the supports of $\mu_{n}$ is bounded (since a sequence converging in the Hausdorff metric is bounded), we infer that $\mu_{n}$ converge weakly to a Borel probability measure $\mu$ whose support is contained in $E$ and $d\left(\mu, \mu_{n}\right) \leq \sum_{k=n+1}^{\infty} d_{k} / 2 \leq d_{n+1}$.

We show that $\mu=\mathcal{H}^{1}\llcorner E$ and that $E$ is AD-regular. For this we estimate the $\mu$ measure of arbitrary sets from above and of disks centered in $E$ from below. 
Suppose that $A$ is a bounded set meeting $E$ and $d_{n} / 2 \leq \operatorname{diam}(A)<d_{n-1} / 2$. Letting $t:=2^{-n} d_{n}$, we observe that in the case when $n$ is even, $E_{n} \cap\{x: \operatorname{dist}(x, A)<$ $t\}$ is contained in at most one of the intervals from $\mathcal{I}_{n-1}$ and in the case when $n$ is odd, it is contained in at most two of the intervals from $\mathcal{I}_{n}$. Thus we conclude from the first statement of Lemma 2.1 (3.1) and from the definition of $\mu_{n}$ that

$$
\begin{aligned}
\mu(A) & \leq \mu_{n}\{x: \operatorname{dist}(x, A)<t\}+d\left(\mu, \mu_{n}\right) / t \\
& \leq \operatorname{diam}(A)+2 t+d_{n+1} / t \leq\left(1+2^{-n+3}\right) \operatorname{diam}(A) .
\end{aligned}
$$

By Besicovitch's density estimate (see [7, Remark 6.3(3)]) this implies that $\mu \leq$ $\mathcal{H}^{1}\llcorner E$.

Let $r_{n}:=2^{-n-9} d_{n}$ and, for each $J=[x, y] \in \mathcal{I}_{n}$, let

$$
J^{*}=\left[x+2^{-n-9}(y-x), y-2^{-n-9}(y-x)\right] .
$$

Also let $E_{n}^{*}=\bigcup_{J \in \mathcal{I}_{n}} J^{*}, F_{k}=\bigcap_{n=k}^{\infty}\left\{z \in E: \operatorname{dist}\left(z, E_{n}^{*}\right) \leq 2 d_{n+1}\right\}$ and $F=$ $\bigcup_{k=1}^{\infty} F_{k}$. Since the set $\left\{z \in E: \operatorname{dist}\left(z, E_{n}^{*}\right)>2 d_{n+1}\right\}$ is covered by the disks $B\left(x, r_{n}+2 d_{n+1}\right)$ and $B\left(y, r_{n}+2 d_{n+1}\right)$ where $J=[x, y] \in \mathcal{I}_{n}$, we have by (3.2)

$$
\mu\left\{z \in E, \operatorname{dist}\left(z, E_{n}^{*}\right)>d_{n}\right\} \leq \sum_{I \in \mathcal{I}_{n}} 20\left(r_{n}+2 d_{n+1}\right) .
$$

Since $\sum_{n} \sum_{I \in \mathcal{I}_{n}} 20\left(r_{n}+2 d_{n+1}\right)<\infty$, we infer from the Borel-Cantelli Lemma that $\mu(E \backslash F)=0$.

If $x \in E$ and $d_{n} \leq r<d_{n-1}$, we find a point $y \in E_{n}$ such that $|x-y| \leq 2 d_{n+1}$ and use the first statement of Lemma 2.1] with $t=2^{-n} d_{n}$ to estimate $\mu(B(x, r)) \geq$ $\mu\left(B\left(y, r-2 d_{n+1}\right)\right) \geq \mu_{n}\left(B\left(y, r-2 d_{n+1}-t\right)\right)-d\left(\mu, \mu_{n}\right) / t \geq r / 5$. In the case when $n$ is even, $x \in F_{n}$ and $r=r_{n}$, we choose $y \in E_{n}^{*}$ and observe that the intersection $B\left(y, r-2 d_{n+1}-t\right) \cap E_{n}$ is a segment of length at least $2\left(r-2 d_{n+1}-t\right)$, and so $\mu(B(x, r)) \geq 2\left(1-2^{-n-1}\right) r$. It follows that $\limsup _{r \rightarrow 0} \mu(B(x, r)) / 2 r \geq 1$ for $\mathcal{H}^{1}$ almost every $x$, which by Besicovitch's density estimate (see [7, Theorem 6.9(2)]) implies that $\mu \geq \mathcal{H}^{1}\llcorner E$.

To recapitulate, we now know that $\mu=\mathcal{H}^{1}\llcorner E$ and that $E$ is AD-regular: one inequality follows from (3.2) and the other from the previous paragraph.

It is easy to see that the orthogonal projection of $E$ to the real axis has measure zero. The same holds for the orthogonal projection of $E$ to the imaginary axis: since $\operatorname{dist}\left(E, E_{n}\right) \leq 2 d_{n+1}$, the projection is covered by intervals of length $4 d_{n+1}$ centered in the (at most $2^{n}$ ) points of the projection of $E_{n}$, so the measure in question is bounded by $2^{n+2} d_{n+1}$, which tends to zero by (3.1). This means that $E$ is purely unrectifiable.

Suppose now that a kernel $K$ satisfies (2.1)-(2.4). Denote $\mu_{\infty}:=\mu$ and

$$
f_{n}(u, s, S):=\int_{A(u, s, S)} K(u-z) d \mu_{n}(z)
$$

for $u \in \mathbb{C}, 0<s \leq S<\infty$ and $n=1,2, \ldots, \infty$.

We show that

$$
f_{n}(y, s, S)=0 \text { provided that } y \in E_{n}^{*} \text { and } 0<s<S \leq r_{n-1} .
$$

Indeed, if $n$ is even, this is obvious since (2.3) implies that the integrand is zero. If $n$ is odd, the set $A(y, s, S) \cap E_{n}$ consists of (one or two) line segments parallel to the real axis and symmetric about the line $\operatorname{Re}(z)=\operatorname{Re}(y)$, so (2.4) gives that $f_{n}(y, s, S)=-f_{n}(y, s, S)$, and we have again that $f_{n}(y, s, S)=0$. 
We let $t_{n}:=2^{-n-9} r_{n}^{2}$ and observe that the fast increase of $m_{k}$ guarantees that

$$
d_{n+1} \leq 2^{-n-4} t_{n} r_{n}
$$

We also note that for every $x$,

$$
\mu_{n}\left(A\left(x, s-t_{n}, s+t_{n}\right)\right) \leq 2^{-n-5} r_{n} \text { provided that } r_{n} \leq s \leq r_{n-1} .
$$

This follows immediately by observing that $A\left(x, s-t_{n}, s+t_{n}\right) \cap E_{n}$ is contained in at most two line segments of total length not exceeding $8 \sqrt{s t_{n}} \leq 2^{-n-5} r_{n}$.

If $y \in E_{n}^{*}, x \in \mathbb{C},|x-y| \leq 2 d_{n+1}$ and $r_{n} \leq s<S \leq r_{n-1}$, then, by (2.1) and (2.2), $|K(x-z)-K(y-z)| \leq C s^{-2}|x-y|$ for $z \in A(x, s, S) \cap A(y, s, S),|K(x-z)| \leq C s^{-1}$ for $z \in A(x, s, S) \backslash A(y, s, S)$ and $|K(y-z)| \leq C s^{-1}$ for $z \in A(y, s, S) \backslash A(x, s, S)$. Hence 3.4 and 3.5 imply that

$$
\begin{aligned}
\left|f_{n}(x, s, S)-f_{n}(y, s, S)\right| \leq & C s^{-2}|x-y| \mu_{n}(A(x, s, S) \cap A(y, s, S)) \\
& +C s^{-1} \mu_{n}(A(x, s, S) \backslash A(y, s, S)) \\
& +C s^{-1} \mu_{n}(A(y, s, S) \backslash A(x, s, S)) \\
\leq & 2^{-n-2} C .
\end{aligned}
$$

Using also (3.3), we infer that

$$
\left|f_{n}(x, s, S)\right| \leq 2^{-n-2} C \text { if } \operatorname{dist}\left(x, E_{n}^{*}\right) \leq 2 d_{n+1} \text { and } r_{n} \leq s<S \leq r_{n-1} .
$$

We now estimate $f_{\infty}(x, s, S)$ for $r_{n} \leq s<S \leq r_{n-1}$ and $\operatorname{dist}\left(x, E_{n}^{*}\right) \leq 2 d_{n+1}$. For this, note that by (2.1) and 2.2) the function $h: A(x, s, S) \rightarrow \mathbb{C}$ defined by $h(z)=K(x-z)$ satisfies $\operatorname{Lip}(h) \leq C / s^{2}$ and $\|h\|_{A(x, s, S)} \leq C / s$. Hence by the second statement of Lemma 2.1 with $t=t_{n},(3.6),(3.5)$ and (3.4)

$$
\begin{aligned}
\left|f_{\infty}(x, s, S)\right| & \leq\left|f_{n}(x, s, S)\right|+\left(C / s^{2}+4 C / s t_{n}\right) d\left(\mu, \mu_{n}\right)+8 C 2^{-n-5} r_{n} / s \\
& \leq 2^{-n} C .
\end{aligned}
$$

We are now ready for the final part of the proof: suppose that $\varepsilon>0$ and $x \in F$. Find an index $q$ such that $x \in F_{q}$ and $2^{-q}<\varepsilon / 2 C$. Whenever $0<r<R<r_{q}$, we choose the largest integer $m$ such that $R \leq r_{m}$ and the smallest integer $k$ such that $r \geq r_{k}$, denote $s_{n}=r_{n}$ for $m<n \leq k, s_{m}=R$ and $s_{k+1}=r$, and use (3.7) to estimate

$$
\left|f_{\infty}(x, r, R)\right| \leq \sum_{n=m}^{k+1}\left|f_{\infty}\left(x, s_{n+1}, s_{n}\right)\right| \leq \sum_{n=m}^{\infty} 2^{-n} C<\varepsilon .
$$

Since $\mu=\mathcal{H}^{1}\left\llcorner E\right.$, this shows that with our choice of $m_{k}$ the principal values associated with any kernel $K$ satisfying (2.1)-(2.4) exist for every $x \in F$, hence $\mathcal{H}^{1}$ almost everywhere on $E$, which concludes the proof of Theorem 3.1 .

As a consequence of [10, Theorem 2.2], we get immediately the following $L^{2}$ boundedness result. The result in [10] is based on the $T(b)$-theorem in 9$]$. (In fact, the result in [10] is stated only for the Cauchy kernel, but the proof given there works for our kernels as well.)

Corollary 3.8. There exists a set $F \subset E$ with a positive $\mathcal{H}^{1}$ measure such that the condition (1.1) holds for $F$. 


\section{ACKNOWLEDGEMENTS}

I would like to express my sincere gratitude to David Preiss for his continuous and extremely valuable support during this work; in fact, the details of the above proof of the theorem and the formulation of the construction are mostly from him. Thanks are also due to Robert Scott, Toby O'Neil and Dean Ives for their comments on linguistics and style. For hospitality I am indebted to the Department of Mathematics at University College London.

\section{REFERENCES}

1. A. P. Calderón, Cauchy integrals on Lipschitz curves and related operators, Proc. Nat. Acad. Sci. U.S.A. $\mathbf{7 4}$ 1977, pp. 1324-1327. MR 57:6445

2. M. Christ, Lectures on Singular Integral Operators, Regional Conference Series in Mathematics 77, Amer. Math. Soc., 1990. MR 92f:42021

3. M. Christ, $A T(b)$ theorem with remarks on analytic capacity and Cauchy integral, Colloq. Math. 60/61 1990, pp. 601-628. MR 92k:42020

4. G. David, Unrectifiable 1-sets have vanishing analytic capacity, to appear in Rev. Mat. Iberoamericana 14 1998, pp. 369-479. MR 99i:42018

5. G. David, P. Mattila, Removable sets for Lipschitz harmonic functions in the plane, Rev. Mat. Iberoamericana 16 2000, pp. 137-215. CMP 2000:15

6. G. David, S. Semmes, Analysis of and on Uniformly Rectifiable Sets, Surveys andf Monographs 38, Amer. Math. Soc., 1993. MR 94i:28003

7. P. Mattila, Geometry of Sets and Measures in Euclidean Spaces, Cambridge University Press, 1995. MR 96h:28006

8. P. Mattila, M. S. Melnikov, J. Verdera, The Cauchy integral, analytic capacity, and uniform rectifiability, Ann. of Math. 144 1996, pp. 127-136. MR 97k:31004

9. F. Nazarov, S. Treil, A. Volberg, Pulling ourselves up by the hair, Preprint.

10. X. Tolsa, Principal values for the Cauchy integral and rectifiability, Proc. Amer. Math. Soc. 128 2000, pp. 2111-2119. CMP 2000:14

Department of Mathematics, University of Jyväskylä, P.O. Box 35, FIN-40351 JYVÄSKYlä, Finland

E-mail address: pjh@math.jyu.fi 